\title{
Network Pharmacology Analysis of Orally Bioavailable SARS-CoV-2 Protease Inhibitor Shows Synergistic Targets to Improve Clinical Efficacy
}

\author{
Arun HS Kumar
}

\begin{abstract}
Introduction: Orally bioavailable SARS-CoV-2 antiviral drugs will significantly improve the clinical management of the disease. PF07321332 (PF32) one such orally bioavailable SARSCoV-2 protease inhibitor which can be helpful to prevent viral replication in the host. Materials and Methods: Hence this study evaluated the network pharmacology of PF32 using established methods to predict its potential safety and efficacy. Results: PF32 was selective against SARS-CoV-2 proteases without any affinity against SARS-CoV-2 RNA polymerase or its spike protein. While PF32 showed pharmacologically relevant affinity against several targets in human tissues. The target profiling of PF32 indicated a fourfold selectivity towards several proteases in human tissues with an affinity $\left(\mathrm{IC}_{50}\right)$ ranging from 26 to $41 \mathrm{nM}$. Conclusion: The predicted inhibitory effects of PF32 against both host and viral proteases may have synergistic effects for superior clinical efficacy.
\end{abstract}

Key words: Molecular interaction, Protease inhibitor, SARS-CoV-2, Antiviral, Coronavirus.

\section{INTRODUCTION}

The pandemic caused by Coronavirus (SARS-CoV-2) which originated in the Wuhan region of China has caused over 150 million human infections (COVID19) globally with a mortality rate of $\sim 2 \%{ }^{1-4}$ Several measures to treated the COVID-19 continues to be explored amid the spread of various mutants collateral to the mass vaccination efforts. One such approach is the development of drugs to inhibit SARS-CoV-2 replication by targeting its protease ${ }^{4-7}$ Several synthetic and natural compounds have shown variable efficacy against SARS-CoV-2 protease. Recently PF07321332 (PF32) is reported as an orally bioavailable protease inhibitor and is currently in clinical trials (Clinical trial ID NCT04756531; http://www.clinicaltrials.gov) to evaluate its safety and efficacy in the treatment of SARS-CoV-2 infections. PF32 specifically inhibits SARS-CoV-2 replicase polyprotein $1 \mathrm{ab}$ which is a multifunctional protein essential to transcription and replication of the coronavirus. ${ }^{3,8} 9$ The orally bioavailability of PF32 will be major advantage in the clinical management of COVID-19. Like all drugs, PF32 may also have off target effects. Understanding of these off targets is essential to envisage potential synergistic and/or adverse effects. Hence in this study network pharmacology of PF32 was assessed using well established tools to get an insight into the all potential targets of PF32 in human cells.

\section{MATERIALS AND METHODS}

\section{Drug structure and target analysis}

The structure of PF32 (Figure 1A) was reconstructed in Swiss target prediction database (http://www. swisstargetprediction.ch) using its SMILES identity. ${ }^{10,11}$ The database was searched for the all human specific targets of PF32 and the probability scores of the targets were analysed.

\section{Protein structure and molecular docking} analysis

The protein data bank (PDB; https://www.rcsb.org) was searched for the $3 \mathrm{D}$ structures of identified targets of PF32 and the data was processed as reported before. ${ }^{7,12}$ The PDB file of PF32 was generated using the Chimera software and used for the analysis of its molecular interactions (number of hydrogen bonds) with all its identified targets as reported before. ${ }^{12-15}$ The reported affinity of homologous structure of PF32 with Fibroblast activation protein alpha (PDB ID IZ68) was used as reference and the affinity of the PF32 with its identified targets was predicted based on the differences in the ratio of hydrogen bonds compared to that of the reference standard as reported before. ${ }^{7,12,16}$ In similar lines the affinity of PF32 against SARS-CoV-2 targets was also estimated as reported before. ${ }^{12,16}$ 


\section{Expression of PF32 targets in human tissues}

Protein expression of PF32 targets in various human tissues was assessed from the human protein atlas database (https://www.proteinatlas.org) on $6^{\text {th }}$ May 2020 as described before. ${ }^{17,18}$

\section{RESULTS}

A majority (49\%) of the PF32 targets in humans tissues are proteases, which is followed by electrochemical transporters (12\%) and family A $\mathrm{G}$ protein coupled receptors (11\%) (Figure 1B). The in silico analysis of the PF32 structure (Figure 1A), indicated several drug/lead like features including its oral bioavailability (Figure 1C). In the swiss target prediction analysis, 19 proteins showed probability scores ranging from 0.11- 0.12 (Figure 1D), which will be referred to as identified targets of PF32. These identified targets were further analysed for their molecular interactions (hydrogen bonds) with PF32 in Chimera software. The number of hydrogen bonds (Figure 1E, 2A) between PF32 and its identified targets did not correlate with their respective probability scores, suggesting other molecular interaction (probably Van der Waals forces) may also influence these interactions. The number of hydrogen bonds between PF32 and its identified targets ranged from 0 to 178 (Figure 1E). Previous reports have indicated an affinity $\left(\mathrm{IC}_{50}\right.$ ) of $73.2 \pm 0.5$ between PF32 homologue and Fibroblast activation protein alpha (FAP) and this was used as a reference to predict the affinity of PF32 with its identified targets (Figure 1f). Affinity $\left(\mathrm{IC}_{50}\right)$ of PF32 against the various receptors ranged from 26 to $4745 \mathrm{nM}$.

The following proteins showed higher affinity (4UFA, 1XU9, 3DDU, $1 \mathrm{H} 8 \mathrm{D}, 1 \mathrm{DUZ}, 2 \mathrm{RA} 3)$ with their $\mathrm{IC}_{50}$ values ranging from 26 to $41 \mathrm{nM}$

\begin{tabular}{|c|c|c|}
\hline PF32 Targets (Common Name) & PDB ID & $\mathrm{IC}_{50}(\mathrm{nM})$ \\
\hline Fibroblast activation protein alpha (FAP) & $1 \mathrm{Z} 68$ & $73.2 \pm 0.5$ \\
\hline Dipeptidyl peptidase IV (DPP4) & $4 \mathrm{~A} 5 \mathrm{~S}$ & $4745 \pm 20.2$ \\
\hline Dipeptidyl peptidase VIII (DPP8 & $6 \mathrm{EOP}$ & NA \\
\hline Prolyl endopeptidase (PREP) & $3 \mathrm{DDU}$ & $34.4 \pm 0.2$ \\
\hline Dipeptidyl peptidase IX (DPP9) & $6 \mathrm{EOR}$ & NA \\
\hline $\begin{array}{l}\text { Baculoviral IAP repeat-containing protein } 2 \\
\text { (BIRC2) }\end{array}$ & $4 \mathrm{HY} 4$ & $79.1 \pm 0.4$ \\
\hline Leukocyte elastase (ELANE) & $5 \mathrm{ABW}$ & NA \\
\hline $\begin{array}{l}\text { HLA class I histocompatibility antigen A-3 } \\
\text { (HLA-A) }\end{array}$ & $1 \mathrm{DUZ}$ & $35.4 \pm 0.1$ \\
\hline Thyrotropin-releasing hormone receptor (TRHR) & NR & $\mathrm{CBE}$ \\
\hline Angiotensin-converting enzyme (ACE) & 4UFA & $26.7 \pm 0.1$ \\
\hline Plasma kallikrein (KLKB1) & $6 \mathrm{O} 1 \mathrm{G}$ & NA \\
\hline Thrombin (F2) & $1 \mathrm{H} 8 \mathrm{D}$ & $34.6 \pm 0.2$ \\
\hline Trypsin I (PRSS1) & 2RA3 & $41.3 \pm 0.4$ \\
\hline $\begin{array}{l}\text { 11-beta-hydroxysteroid dehydrogenase } 1 \\
\text { (HSD11B1) }\end{array}$ & 1XU9 & $28.6 \pm 0.1$ \\
\hline Inhibitor of apoptosis protein 3 (XIAP) & $4 \mathrm{~J} 44$ & $158.2 \pm 1.2$ \\
\hline $\begin{array}{l}\text { 11-beta-hydroxysteroid dehydrogenase } 2 \\
\text { (HSD11B2) }\end{array}$ & NR & $\mathrm{CBE}$ \\
\hline Pepsinogen C (PGC) & 1HTR & $677.9 \pm 4.1$ \\
\hline Dipeptidyl peptidase II (DPP7) & $4 \mathrm{EBB}$ & NA \\
\hline TRAIL receptor-1 (TNFRSF10A) & $5 \mathrm{CIR}$ & $197.7 \pm 1.6$ \\
\hline
\end{tabular}

NR: not reported. NA: no affinity, CBE: cannot be estimated.

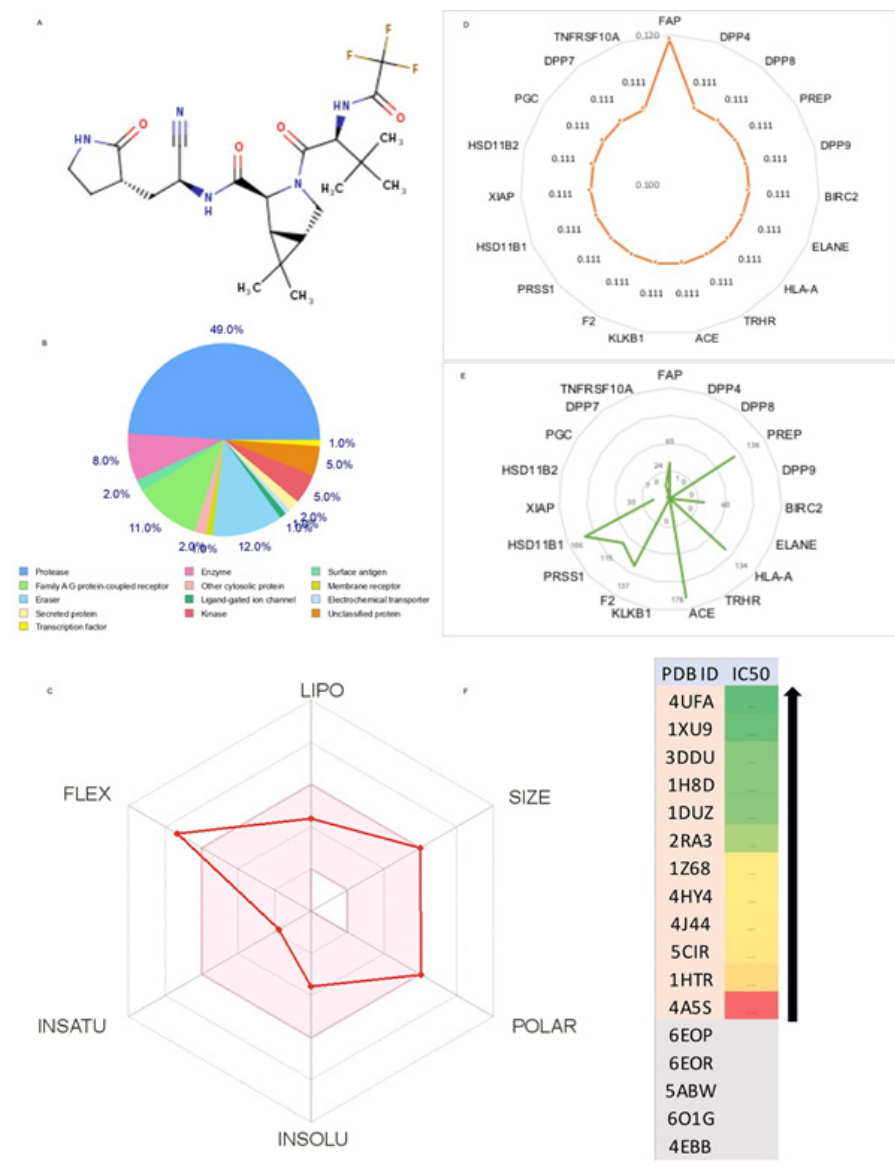

Figure 1: Pharmacological properties of PF07321332 (PF32). A) Structure of PF32. B) Summary of identified targets of PF32 in humans. C) Drug and oral bioavailability properties of PF32 based on its size, lipophilicity (LIPO), structure flexibility (FLEX), polarity, instauration (INSATU) and solubility (INSOLU). D) Probability of interaction score of identified targets of PF32 in humans. E) Number of hydrogen bonds between PF32 and its identified targets at $10 \AA$ distance. F) Affinity $\left(\mathrm{IC}_{50}\right)$ of PF32 against its identified targets (direction of arrow is from low to high affinity, targets in grey have no affinity).

(Table 1). PF32 showed higher affinity with Identified targets 4UFA and 1XU9 and least affinity with 4A5S (Table 1). Nevertheless the affinities predicted with 11 of the Identified targets were in pharmacologically relevant concentrations (Table 1).

The affinity of PF32 against various SARS-CoV-2 specific targets were also assessed. Representative images of molecular interaction of PF32 against selected identified and SARS-CoV-2 specific targets are shown in Figure 2A. PF32 showed highest affinity ( $\left.\mathrm{IC}_{50} 45-60 \mathrm{nM}\right)$ against SARS-CoV-2 main protease and Replicase polyprotein 1ab (Figure 2B). As several 3D structures of Replicase polyprotein $1 \mathrm{ab}$ are reported in the PDB databased, a representative of each of the variable 3D structure reported was screened in this study. PF32 did not show any affinity against SARS-CoV-2 spike protein, RNA polymerase and some variable structures of Replicase polyprotein $1 \mathrm{ab}$ (Table 2).

The expression pattern of the identified targets of PF32 was evaluated in the human tissues. Expression pattern of representative identified targets (ACE, HSD11B1, PREP and FAP) are shown in Figure 3. While some identified targets were expressed only in selected tissues (ACE and HSD11B1) others were ubiquitously expressed (PREP and FAP) (Figure 3). 


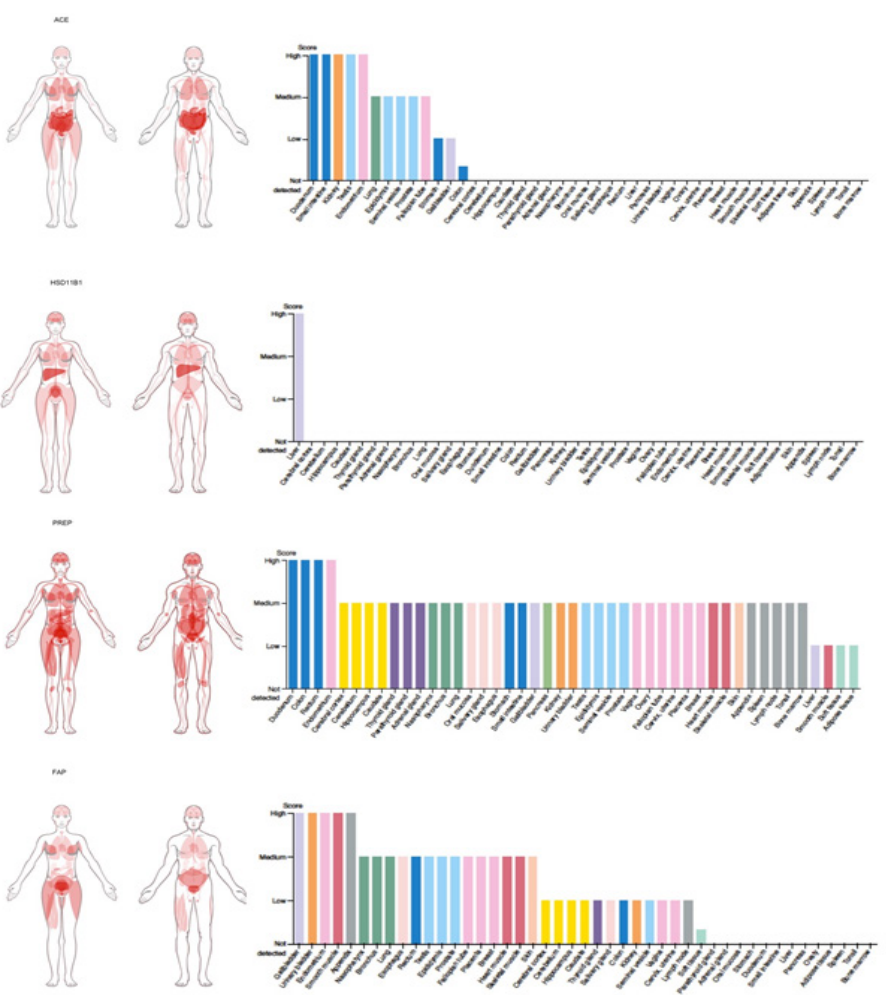

Figure 3: Anatomogram of selected targets of PF32 in human tissues. The graphs show the expression levels of the respective protein in various humans tissues.

Table 2: Affinity of PF32 against SARS-COV-2 targets.

\begin{tabular}{|c|c|c|}
\hline Target Type & PDB ID & $\mathrm{IC}_{50}(\mathrm{nM})$ \\
\hline Main protease & $6 Y 84$ & $48.9 \pm 1.2$ \\
\hline Spike protein & $6 \mathrm{VSB}$ & NA \\
\hline RNA polymerase & $6 \mathrm{M} 71$ & NA \\
\hline 3C-like protease & 7JPY & $206.3 \pm 8.4$ \\
\hline Replicase polyprotein 1ab & $5 \mathrm{R} 82$ & $58.6 \pm 1.4$ \\
\hline Replicase polyprotein 1ab & $5 \mathrm{RFC}$ & $59.3 \pm 1.2$ \\
\hline Replicase polyprotein $1 \mathrm{ab}$ & 5RF9 & $59.3 \pm 1.3$ \\
\hline Replicase polyprotein 1ab & $5 \mathrm{RGJ}$ & $56.5 \pm 1.1$ \\
\hline Replicase polyprotein 1ab & $5 \mathrm{RL} 7$ & NA \\
\hline Replicase polyprotein $1 \mathrm{ab}$ & $5 \mathrm{RS} 7$ & $115.7 \pm 6.4$ \\
\hline Replicase polyprotein 1ab & $5 R V P$ & $63.3 \pm 1.8$ \\
\hline Replicase polyprotein $1 \mathrm{ab}$ & $5 \mathrm{~S} 71$ & NA \\
\hline Replicase polyprotein 1ab & 6W01 & NA \\
\hline Replicase polyprotein $1 \mathrm{ab}$ & $6 \mathrm{X} 4 \mathrm{I}$ & NA \\
\hline Replicase polyprotein 1ab & $6 Y V F$ & $54.5 \pm 1.1$ \\
\hline Replicase polyprotein $1 \mathrm{ab}$ & $7 \mathrm{NFV}$ & $83.2 \pm 2.4$ \\
\hline Replicase polyprotein 1ab & 7LTJ & NA \\
\hline Replicase polyprotein $1 \mathrm{ab}$ & 7KYU & $65.9 \pm 2.1$ \\
\hline Replicase polyprotein 1ab & 7JYC & NA \\
\hline Replicase polyprotein $1 \mathrm{ab}$ & 7D1M & NA \\
\hline Replicase polyprotein $1 \mathrm{ab}$ & $6 Z S L$ & NA \\
\hline
\end{tabular}

NA: no affinity.
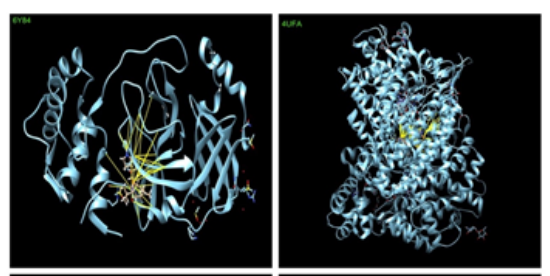

PDBID IC50
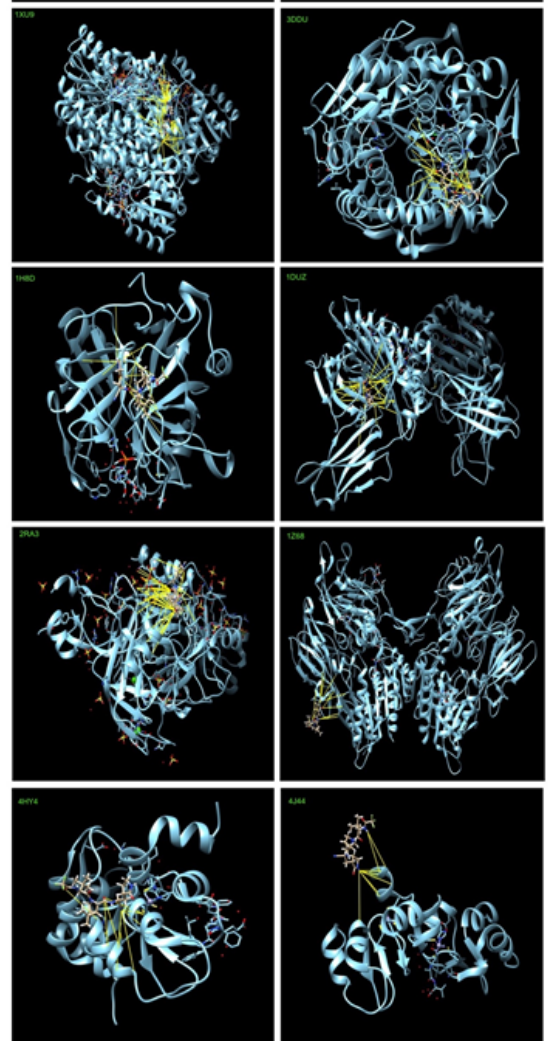

$6 Y 84$

6YVF

5RGJ

5R82

5 RFC

5RF9

5RVP

7KYU

7NFV

5RS7

7JPY

6VSB

6M71

5RL7

$5 \mathrm{~S} 71$

6W01

6X41

7LTJ

7JYC

7D1M

6ZSL

Figure 2: Molecular interactions and affinity of PF07321332 (PF32) with its targets. A) Selected images showing interactions of PF32 with its identified targets in humans and SARS-COV-2 (yellow lines indicate the hydrogen bonds). B) Affinity ( $\left(\mathrm{I}_{50}\right)$ of PF32 against various SARS-COV-2 targets (direction of arrow is from low to high affinity, targets in grey have no affinity).

\section{DISCUSSION}

PF32 is recently developed orally bioavailable SARS-CoV-2 protease inhibitor which has entered clinical safety and efficacy evaluation phase (Clinical trial ID NCT04756531) (https:/go.drugbank.com/drugs/ DB16691). This study reports the network pharmacology analysis to identify human tissue and SARS-CoV-2 specific targets the PF32 molecule can interact with. Knowledge of these interactions will be essential to understand the safety and efficacy of PF32 as a supplement to that identified in clinical trials. ${ }^{19-21}$ The target profiling of PF32 indicated a fourfold selectivity towards proteases with an affinity ( $\mathrm{IC}_{50}: 26$ to 41 $\mathrm{nM}$ ) which was pharmacologically relevant. In the human tissue the affinity of PF32 was maximum towards Angiotensin-converting enzyme (ACE). Considering the reports of SARS-CoV-2 using the ACE2 as receptor for entering into host cell, ${ }^{3,22}$ the affinity of PF32 towards ACE may evince synergistic effects by both inhibiting the virus multiplication as well as preventing virus entry into host cells. Besides ACE, several other proteases were also overserved to have pharmacologically relevant affinity with PF32. Although the clinical relevance of these interactions are unclear at present, considering the systemic inflammation evinced 
by SARS-CoV-2, ${ }^{23-25}$ the broader protease inhibitory potential of PF32 observed in this study may facilitate synergistic clinical benefits.

Similar to the broader affinity of PF32 against several human tissue specific proteases, PF32 was observed to have pharmacologically relevant affinity against SARS-CoV-2 main protease as well as its Replicase polyprotein lab. This selective inhibition of SARS-CoV-2 proteases but not its RNA polymerase or spike protein with higher affinity $\left(\mathrm{IC}_{50}: 45\right.$ to $\left.60 \mathrm{nM}\right)$ together with its bioavailability/cell permeability may potentiate clinical safety and efficacy of PF32. However unlike the SARS-CoV-2 targets, the identified targets of PF32 in human tissues had a wider tissue expression profile, which paraphs reflects the wider pharmacological profile of PF32. Depending on the tissue specific virus presence and considering associated systemic inflammation the diffused pharmacological profile of PF32 may prove to be clinically beneficial.In summary the network pharmacology analysis of PF32 in this study identifies its relevant targets in human tissues and SARS-CoV-2, which may have synergistic effects for superior clinical efficacy.

\section{ACKNOWLEDGEMENT}

Research support from University College Dublin-Seed funding/Output Based Research Support Scheme is acknowledged.

\section{CONFLICT OF INTEREST}

The author declares that there is no conflict of interest.

\section{REFERENCES}

1. Cucinotta D, Vanelli M. WHO Declares COVID-19 a Pandemic. Acta Biomed. 2020;91(1):157-60

2. Fauci AS, Lane HC, Redfield RR. Covid-19 - Navigating the Uncharted. N Engl J Med. 2020;382(13):1268-9.

3. Stower H. Virological assessment of SARS-CoV-2. Nat Med. 2020;26(4):465.

4. Kilroy D, Kumar A. Anatomical perspective on the loss of smell and taste sensation in SARS-CoV-2 infection. . Anatomy. 2020;14(2):145-9.

5. Kumar AHS. Pharmacology of Chloroquine: Potential Mechanism of Action against Coronavirus. BEMS Reports. 2020;6(1):9-10.

6. Kumar AHS, Sharma V. Acetamido-Propanoic Acid Derived Compounds as Protease Inhibitors to Target Coronaviruses. BEMS Reports. 2019;5(2):20-2.

7. Sagar VK, Kumar AHS. Efficacy of Natural Compounds from Tinospora cordifolia against SARS-CoV-2 Protease, Surface Glycoprotein and RNA Polymerase. BEMS Reports. 2020;6(1):6-8.

8. Beck BR, Shin B, Choi Y, et al. Predicting commercially available antiviral drugs that may act on the novel coronavirus (SARS-CoV-2) through a drug-target interaction deep learning model. Comput Struct Biotechnol J. 2020;18:784-90.

9. Choy KT, Wong AY, Kaewpreedee P, et al. Remdesivir, lopinavir, emetine, and homoharringtonine inhibit SARS-CoV-2 replication in vitro. Antiviral Res. 2020;178: 104786.

10. Gfeller D, Grosdidier A, Wirth M, et al. Swiss Target Prediction: A web server for target prediction of bioactive small molecules. Nucleic Acids Res. 2014;42(Web Server issue):W32-8.

11. Daina A, Michielin O, Zoete V. Swiss Target Prediction: Updated data and new features for efficient prediction of protein targets of small molecules. Nucleic Acids Res. 2019;47(W1):W357-64.

12. Kumar AHS. Molecular profiling of Neprilysin expression and its interactions with SARS-CoV-2 spike proteins to develop evidence base pharmacological approaches for therapeutic intervention., Research Square, Online. 2021;1-16.

13. Goothy SSK, Kumar AHS. Network Proteins of Angiotensin-converting Enzyme 2 but Not Angiotensin-converting Enzyme 2 itself are Host Cell Receptors for SARS-Coronavirus-2 Attachment. BEMS Reports. 2020;6(1):1-5.

14. Kumar AHS. Molecular Docking of Natural Compounds from Tulsi (Ocimum sanctum) and neem (Azadirachta indica) against SARS-CoV-2 Protein Targets. BEMS Reports. 2020;6(1):11-3.

15. Yang Z, Lasker K, Schneidman-Duhovny D, et al. UCSF Chimera, MODELLER, and IMP: An integrated modeling system. J Struct Biol. 2012;179(3):269-78.

16. Kumar A. Modelling the efficacy of Neprilysin from various species in degrading different Amyloid-beta peptides: Potential application in therapeutics of Alzheimers disease. Bio Rxiv. 2021;1-16.

17. Hruz T, Laule O, Szabo G, et al. Genevestigator v3: A reference expression database for the meta-analysis of transcriptomes. Adv Bioinformatics. 2008;2008:420747.

18. Uhlen M, Fagerberg L, Hallstrom BM, et al. Proteomics. Tissue-based map of the human proteome. Science. 2015;347(6220):1260419.

19. Casas Al, Hassan AA, Larsen SJ, et al. From single drug targets to synergistic network pharmacology in ischemic stroke. Proc Natl Acad Sci U S A. 2019;116(14):7129-36

20. Khanal P, Patil BM. Integration of network and experimental pharmacology to decipher the antidiabetic action of Duranta repens L. J Integr Med. 2021:19(1):66-77.

21. Khanal P, Duyu T, Patil BM, et al. Screening of JAK-STAT modulators from the antiviral plants of Indian traditional system of medicine with the potential to inhibit 2019 novel coronavirus using network pharmacology. 3 Biotech. 2021;11(3):119.

22. Li W, Moore MJ, Vasilieva N, et al. Angiotensin-converting enzyme 2 is a functional receptor for the SARS coronavirus. Nature. 2003:426(6965):450-4.

23. Katopodis P, Kerslake R, Davies J, et al. COVID19 and SARSCoV2 host cell entry mediators: Expression profiling of TMRSS4 in health and disease. Int $\mathrm{J} \mathrm{Mol}$ Med. 2021;47(4):1-7.

24. Shtaya A, Trippier S, Ghatala R, et al. Comment on "Stroke in patients with SARSCoV2 infection: Case series" from a London hospital experience. J Neurol. 2021;268(2):424-30.

25. Mandel M, Harari G, Gurevich M, et al. Cytokine prediction of mortality in COVID-19 patients. Cytokine. 2020;134:155190. 